\title{
Writer Identification Using Super Paramagnetic Clustering and Spatio Temporal Neural Network
}

\author{
Seyyed Ataollah Taghavi Sangdehi and Karim Faez \\ Qazvin Azad University of Iran, +98-911-155-5819 \\ Amirkabir University, Tehran, Iran \\ atataghavi@gmail.com, kfaez@aut.ac.ir
}

\begin{abstract}
This paper discusses use of Super Paramagnetic Clustering (SPC) and Spatio Temporal Artificial Neuron in on-line writer identification, on Farsi handwriting. In online cases, speed and automation are advantages of one method on others, therefore we used unsupervised and relatively quick clustering method, which in comparison with conventional approaches, give us better result. Moreover, regardless of various parameters that available from acquisition systems, we only consider to displacement of pen tip at determined direction that lead to quick system due to its quick preprocessing and clustering. Also we use a threshold that remove displacement between disconnected point of a word that lead to a better classification result on on-line Farsi writers.
\end{abstract}

Keywords: Writer Identification, Super Paramagnetic Clustering, Spatio Temporal Neural Network.

\section{Introduction}

With the rapid development of the information technology, true user authentication, using biometrics information will be required to get the more reliable password. the necessity of person identification is increasing for example, in bank, shop, ecommerce and so on. In this work, person identification using handwriting, is referred to as writer identification. the target of writer identification is to quest for the personal identity, among a group of possible candidates, which is mainly used in securityoriented applications.

Writer identification problem is largely classified into two classes. One is offline methods, based on only static visual information, and the other is, on-line methods, based on dynamics of the handwriting process. A major advantage of the later method is very difficult to forge or copy the invisible dynamic features. We can say writer identification is identifying, writer from a written script such as character, signature and etc. Many methods of on-line signature recognition have been proposed at [1], [2], [3]. In the online methods, dynamic features of handwriting process have been used, such as pen point coordination [4], writing velocity [5], azimuth [6] and other features which are available from a digitizer. Due to the seemingly uniqueness of physiological and behavioral characteristics of each individual, writer identification has shown [1] to be a feasible task. Each writer's writing, has a set of characteristics which is exclusive to him, only. 
However a few methods for on-line writer identification (exclusively, handwriting and not signature) have been presented. It is known that Farsi handwriting (words) consist of several stroks and are different from continuous English handwriting.

In this paper, we propose a novel approach for on-line writer identification based on Super Paramagnetic Clustering (SPC) algorithm [9] and Spatio Temporal Artificial Neuron (STAN) [10],[11] on Farsi handwriting. The rest of this paper is organized as follows. Section 2 gives a short overview of the SPC. Section 3 describes the writer identification procedure based on the SPC clustering and STAN classification. Experimental results and conclusion are provided in Section 4.

\section{Overview of Super Paramagnetic Clustering}

The key idea of Super Paramagnetic Clustering (SPC) is based on magnetic property of material at different temperature. Each material reach to high magnetic properties at special temperature. We use this temperature as a best value for clustering with SPC. The following is key ideas of Super Paramagnetic Clustering (SPC) [7], which is based on simulated interactions between each point and its $\mathrm{k}$-nearest neighbors.

There are $q$ different states per each magnetic particle. First step is to represent the $m$ selected features of each spike $i$ by a point $\boldsymbol{x}_{i}$ in an $m$-dimensional phase space. The interaction strength between points $\boldsymbol{x}_{i}$ is then defined as:

$$
J_{i j}=\left\{\begin{array}{lc}
\frac{1}{k} \exp \left(-\frac{\left\|x_{i}-x_{j}\right\|^{2}}{2 a^{2}}\right) & \text { If } \boldsymbol{x}_{i} \text { is a nearest neighbor of } x_{j} \\
0 & \text { Else }
\end{array}\right.
$$

where $a$ is the average nearest-neighbors distance and $k$ is the number of nearest neighbors. Note that the strength of interaction $J_{i j}$ between nearest neighbor spikes decays exponentially, with increasing Euclidean distance $d_{i j}=\left\|x_{i}-x_{j}\right\|^{2}$, which corresponds to the similarity of the selected features. In the second step, an initial random state $s$ from 1 to $q$ is assigned to each point $\boldsymbol{x}_{i}$. Then $N$ Monte Carlo iterations are run for different temperatures $T$, given an initial configuration of states $s$. A point $\boldsymbol{x}_{i}$ is randomly selected and its state $s$ changed to a new state $s_{n e w}$, which is randomly chosen between 1 and $q$. probability that, the nearest neighbors of $\boldsymbol{x}_{i}$ will also change their state to $s_{n e w}$ is given by:

$$
P_{i j}=1-\exp \left(-\frac{J_{i j}}{T} \delta_{s_{i}, s_{j}}\right)
$$


Where $T$ is the temperature in which, this probability compute. Note that only those nearest neighbors of $\boldsymbol{x}_{i}$ which were in the same previous state $s$, are candidates to change their states to $s_{n e w}$. Neighbors which change their values, create a frontier and cannot change their state again, during the same iteration. Points which do not change their state in a first attempt, can do so, if revisited during the same iteration. Then for each point of the frontier, we apply equation (2), again to calculate the probability of changing state to $s_{n e w}$ for their respective neighbors. The frontier is updated, and the update is repeated until the frontier does not change any more.

At that stage, we start the procedure again from another point and repeat it several times, in order to get the representative statistics. Points which are close together, (corresponding to a given cluster) change their state together. This can be quantified by measuring the point to point correlation $\delta_{s_{i}, s_{j}}$ and defining $\boldsymbol{x}_{i}$ and $\boldsymbol{x}_{j}$ to be members of the same cluster if $\delta_{s_{i}, s_{j}} \geq \theta$, for a given threshold $\theta$. Clustering results, mainly depend on temperature and are robust to small changes on other parameters, like, threshold, number of nearest neighbors and states [9]. This method remains better results in sorting of spikes in comprise with other approach (Table.1).

\section{Writer Identification Based on SPC and Spatio Temporal Neural Network}

In this section we describe on-line writer identification. We get data from a tablet and apply preprocessing to produce displacement, which is converted to impulses in the form of spatio temporal coding in polar coordinates. Accumulated impulses at a temporal window go to a clustering unit as an input. After clustering, clustered data at another pass of algorithm, can produce impulses, like previous phase. Accumulated impulses from this section are representative of a person who must be identified.

\subsection{Data Acquisition and Preprocessing}

On-line writer identification methods, often use a data convertor device. The user registers his/her own written samples with a special pen, and handwriting, received online. The device we use at this experiment is a tablet from Wacom company, at A4 size, in which the sampling rate is 200 points per second. We record samples of each person in a text file. From the tablet we acquire signals of position coordinates of pen on surface of the tablet. Note that each handwriting word, consists of a sequence of pen tip coordinates. We can use displacement from these positions. Since the beginning of each word can be any point at writing surface, we use displacements at discrete directions [10], [11] which make this system translation invariant. Each displacement take place at one direction, by quantization of direction to nearest basic direction, among 8 defined direction which is the best number of features for input to our proposed method according to experiments. According to Fig.1, we make a vector with dimension equal to number of quantized direction that achieve at our experiment. Therefore we have 8 components. displacements at a direction take place on one of 
these components that can be an impulse at time. There are sequences of displacements at each handwriting. Then we must convert them to sequences of impulses.

This process is a spatio temporal problem. thus each displacement can be an impulse at a given time. We present spatio temporal coding by complex number [10]. Each signal $\mathrm{x}$ at time $\mathrm{t}$ is shown with amplitude $\eta$ and phase $\phi$ (temporal position) from a reference time, at polar coordinate $(\eta, \phi)$

$$
\begin{gathered}
x=\eta e^{i \phi} \text { and } \tan (\phi)=\mu_{t} \tau \\
x=\eta e^{i \arctan \left(\mu_{t} \tau\right)}
\end{gathered}
$$

and because of decreasing amplitude of $\mathrm{x}$ due to time, we have:

$$
\begin{gathered}
x_{i}(t)=\eta e^{-\mu_{s} \tau} e^{-i \arctan \left(\mu_{t} \tau\right)} \\
\mu_{s}=\mu_{t}=\frac{1}{T W} \\
x_{j}\left(t_{2}\right)=\eta_{1} e^{-\mu_{s}\left(t_{2}-t_{1}\right)} e^{-i \arctan \left(\mu_{t}\left(t_{2}-t_{1}\right)\right)}
\end{gathered}
$$

Where $x_{i}$ is an impulse at $i$ th component of 8 dimensional displacement vector at time $t$, and TW is a temporal window in which feature vectors are created. When a new impulse $x_{j}$ is emitted on a given component at time $t_{2}$, it is accumulated with the previous impulse according to (7). We need, creation of primary feature vector for clustering unit and also, feature vector for classification unit, so we consider the suitable temporal window which obtain from data set.

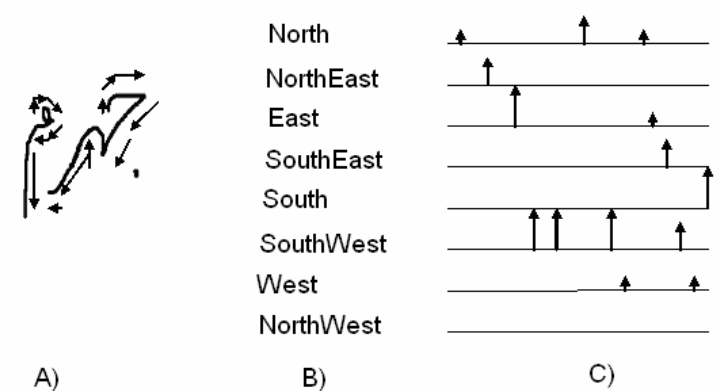

Fig. 1. A) Pen tip displacement at writing a word. B) Quantized direction. C) Sequence of displacement at quantized direction as impulse. 
Preprocessing have an important role in obtaining better results. Preprocessing algorithm used in [11] was suitable for continuous English words. Since for Farsi handwritten word, each word can consist of one or some disjoint part, this algorithm can't work well on Farsi handwriting with disjoint sub words. Sudden jumping of pen tip at disjoint parts of a word that usually occurs between marks of a letter and other strokes of word or occurs between two disjoint sub words of a word (Fig.2) can lead to unuseful features, thus we use a threshold for displacement at defined direction and avoid displacement higher than this threshold.

\subsection{Clustering of Sub Words}

Handwriting, created from sub words that sequence of them, create whole word. We consider for each of sub word, a feature vector which, will be extracted at a defined temporal window. We can define each displacement at quantized direction at time, as an impulse. We convert asynchronous but continuous flow of impulses from preprocessing unit to spatio temporal vectors. These vectors are made using spatio temporal coding and accumulation of impulses at temporal window in preprocessing phase.

Thus each vector saves impulse information (sub word information) at temporal window. Therefore at each period of time, equal to TW, we have a vector, considered as an input to clustering unit.

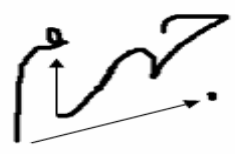

Fig. 2. Sudden jumping from one disjoint sub word to another disjoint sub word. Note that the, arrow line shows sudden jumping.

Temporal window is defined according to experimental results, obtained from dataset. Note that TW is selected so that performance of system does not decrease. Created vectors of this section are representative of a sub word (Fig.3). Sequence of these sub words vector, for each person at defined TW are behavioral identifier for a person. Now we configure SPC algorithm for this application. We get feature vector of preprocessing unit which has 8 dimensions and run SPC for different ranges of temperature $\mathrm{T}$, until finding maxima of $\mathrm{T}$, in which each cluster have at minimum 20 points. To run this algorithm automatically, we use a criterion based on size of clusters. First we define minimum number of data samples which must be in a cluster. This is because that increasing temperature can lead to cluster with a few point in it. In fact at high temperature the number of clusters increases [9], so we can overcome this problem, with size criterion of clusters so that we can define size of a cluster to be fraction of data set. If there isn't enough points in each cluster, we use minimum temperature. With this work we guarantee automation of method (one of advantage over Kmeans clustering) and find optimized temperature. We set parameters of SPC as below according to [9]: number of state $\mathrm{q}=20$, number of nearest neighbor $\mathrm{k}=11$, and 


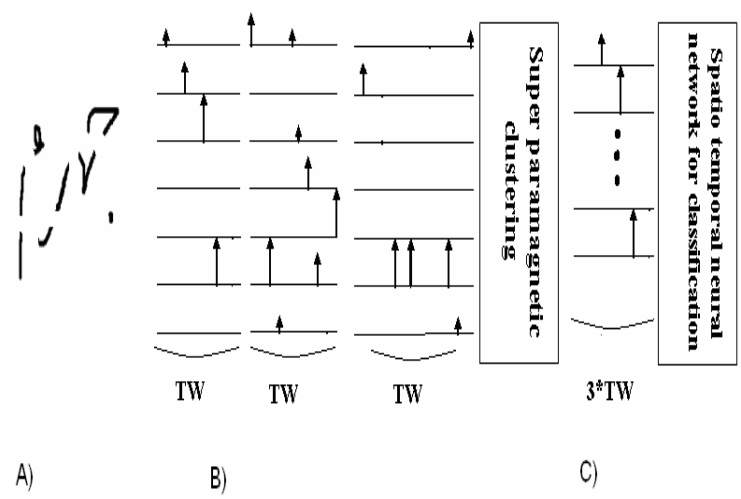

Fig. 3. A) A word includes sequence of sub words. B) Sequence of sub words in form of impulse at determined TW. C) Feature vector of whole word for classification.

also number of iteration $\mathrm{N}=500$ and correlation threshold $\theta=0.5$. Note that changing above parameters does not affect result and classification rate depends on temperature only. Due to each iteration we change temperature $\mathrm{T}$ from 0 to 0.2 by step 0.01 and find maximum temperature in which each cluster included at minimum, 20 points or fraction of dataset that is determined.

\subsection{Handwriting Feature Vector Classification}

Feature vectors of previous section are saved according to the time of occurrence (end time of TW). In this section we can extract feature vectors of classification unit. Because of each handwriting word is a sequence of sub words at specific times, we use spatio temporal coding like preprocessing unit. Then using ST-RCE [10], [11] algorithm which is a spatio temporal neural network, classify feature vectors.

Output vector of clustering units has a dimension equal to number of clusters, that relevant element of this vector with cluster of vector is activated, when a vector of previous section come into clustering unit. Time of this event is the same as the reference time that was saved with vector. Accumulation of signals on a dimension are computed based on a reference time (here, mean sampling time of each word at database) according to (5),(6),(7). Each vector is corresponds to with a handwriting word of a person and each person corresponds to a class. Thus we can classify them using ST_RCE algorithm. This algorithm uses hermitian distance given in (8) or (9) to determine, belonging a vector to a class or not.

$$
\begin{gathered}
V(X, W)=\sum_{j=1}^{n} \overline{w_{j}} x_{j} \\
D(x, w)=\sqrt{\sum_{j=1}^{n}\left(x_{j}-w_{j}\right)\left(x_{j}-w_{j}\right)}
\end{gathered}
$$

Where $\mathrm{D}$ and $\mathrm{V}$ are distances between vector $\mathrm{x}$ and weight of neural network. 


\section{Experiments and Conclusion}

In one experiment we used two databases according to table1. These databases were made using handwritings of 20 persons acquired by a tablet. We asked each person to writes 66 words generally selected from names of cities. We need enough number of samples. Thus we asked each person to write each word 7 times. At the second database for simplicity we use the first database with a little change: we force three persons to try to forge 20 words of handwriting next to original handwriting and next to original person.

We divided database to 5 dataset to train and test. Several algorithms can be used for spike clustering and best of them is an algorithm that has more discriminant strength. In order to show these differences with the proposed method we compare results of SPC clustering with Kmeans [11]. Experimental results (Table.1) shows, in cases that discrimination between spike shapes are relatively easy, two algorithms are the same, but in cases that shapes of spikes are very similar (trying to forge), SPC has acceptable advantage over Kmeans. Moreover, with SPC, we have an fully unsupervised clustering unlike Kmeans that we define number of clusters.

In another experiments we used five databases. We divide our dataset to four databases (Table.2) according to the sudden jumping in each word (Fig.2). With removing unnecessary displacement at sub words by preprocessing (jumping from disjoint sub words to another disjoint sub words of a word) the performance of classifier can increase according to (Table.2).

We found that other features beside to displacement can not increase precision of classification and moreover increase time of classification.

Note that some of the human being behaviors, like, difficulty in writing on special surface or writing with special pen can lead to undesired results, which we encountered them at the data acquisition phase. Therefore the accuracy of data acquisition, can lead to a better classification results. Unfortunately there isn't any standard database at this context and make difficult comprise of result, achieved from various method.

Table 1. Precision of methods on each type of database according to the number of forgeries

\begin{tabular}{cccccc}
\hline & $\begin{array}{c}\text { All } \\
\text { samples }\end{array}$ & Forgeries & With Kmeans[11] & $\begin{array}{c}\text { With } \\
\text { preprocessing }\end{array}$ & $\begin{array}{c}\text { Proposed } \\
\text { method }\end{array}$ \\
\hline Database1 & 9240 & 0 & $71.3 \%$ & $75.2 \%$ & $76.2 \%$ \\
Database2 & 9240 & 420 & $68.2 \%$ & $73.1 \%$ & $79.1 \%$ \\
\hline
\end{tabular}

Table 2. Precision of methods on each databases according to the number of jumping at a word

\begin{tabular}{ccccccc}
\hline & $\begin{array}{c}\text { number of sudden } \\
\text { jumping }\end{array}$ & Samples & With [11] & With SPC & $\begin{array}{c}\text { [11]with } \\
\text { preprocessing }\end{array}$ & $\begin{array}{c}\text { Proposed } \\
\text { method }\end{array}$ \\
\hline Db1 & 1 & $18 * 20 * 7$ & $78.2 \%$ & $82.1 \%$ & $82.6 \%$ & $84.2 \%$ \\
Db2 & 2 & $17 * 20 * 7$ & $77.2 \%$ & $79.1 \%$ & $80.6 \%$ & $81.8 \%$ \\
Db3 & 3 & $12 * 20 * 7$ & $72 \%$ & $76.6 \%$ & $79.4 \%$ & $80.1 \%$ \\
Db4 & 4 & $12 * 20 * 7$ & $69.1 \%$ & $71 \%$ & $73.3 \%$ & $77.3 \%$ \\
& & & & & \\
\hline
\end{tabular}




\section{References}

1. Gupta, S.: Automatic Person Identification and Verification using Online Handwriting, thesis,Hyderabad, INDIA (2008)

2. Faundez-Zanuy, M.: On-line signature recognition based on VQ-DTW. Pattern Recognition 40, 981-992 (2007)

3. Kashi, R., Hu, J., Nelson, W.L.: A Hidden Markov Model approach to online handwritten signature verification. IJDAR 1, 102-109 (1998)

4. Thumwarin, P., Tangtisanon, P., Murata, S., Matsuura, T.: On-line Writer Recognition for Thai Numeral. In: Proc. IEEE Circuits and Systems, Asia-pacific Conference, pp. 503-508 (2002)

5. Thumwarin, P., Matsuura, T.: On-line Writer Recognition for Thai Based on Velocity of Barycenter of Pen-point Movement. In: ICIP, pp. 889-892 (2004)

6. Hangai, S., Yamanaka, S., Hamamoto, T.: On-line Signature Verification Based On Altitude and Direction of Pen Movement. In: ICME, pp. 489-492 (2000)

7. Blatt, M., Wiseman, S., Domany, E.: Super Paramagnetic Clustering of Data. Phys. Rev. Lett. 76, 3251-3254 (1996)

8. Wolf, U.: Comparison Between Cluster Montecarlo algorithm in the Ising spin model. Phys.Lett.B 228, 379-382 (1989)

9. Quiroga, Q., Nadasty, R., Ben-Shaul, Z.: Unsupervised Spike Detection and Sorting With Wavelets and Superparamagnetic Clustering. Neural Computation 16, 1661-1687 (2004)

10. Baig, R.: Spatial-Temporal Artificial Neurons Applied to On-line Cursive Handwritten Character Recognition. In: ESANN, pp. 561-566 (2004)

11. Baig, R., Hussain, M.: On-line Signture Recognition and Writer Identification Using Spatial-Temporal Processing. In: INMIC, pp. 381-385 (2004)

12. Li, B., Zhang, D., Wang, K.: Online signature verification based on null component analysis and principal component analysis. Pattern anal. applic., 345-356 (2005)

13. Zhang, K., Nyssen, E., Sahli, H.: A Multi-Stage Online Signature Verification System. Pattern Analysis \& Application (5), 288-295 (2002) 